\title{
Ion-ion coincidence imaging at high event rate using an in-vacuum pixel detector
}

Jingming Long (龙精明), Federico J. Furch, Judith Durá, Anton S. Tremsin, John Vallerga, Claus Peter Schulz, Arnaud Rouzée, and Marc J. J. Vrakking

Citation: The Journal of Chemical Physics 147, 013919 (2017); doi: 10.1063/1.4981126

View online: $\mathrm{http}: / / \mathrm{dx}$.doi.org/10.1063/1.4981126

View Table of Contents: http://aip.scitation.org/toc/jcp/147/1

Published by the American Institute of Physics

\section{Articles you may be interested in}

A new electron-ion coincidence 3D momentum-imaging method and its application in probing strong field dynamics of 2-phenylethyl-N, N-dimethylamine

The Journal of Chemical Physics 147, 013920 (2017); 10.1063/1.4981526

Time-resolved multi-mass ion imaging: Femtosecond UV-VUV pump-probe spectroscopy with the PImMS camera

The Journal of Chemical Physics 147, 013911 (2017); 10.1063/1.4978923

Coincidence velocity map imaging using a single detector

The Journal of Chemical Physics 147, 013922 (2017); 10.1063/1.4981917

Perspective: Advanced particle imaging

The Journal of Chemical Physics 147, 013601 (2017); 10.1063/1.4983623

Finite slice analysis (FINA)—A general reconstruction method for velocity mapped and time-sliced ion imaging

The Journal of Chemical Physics 147, 013913 (2017); 10.1063/1.4979305

Advantage of spatial map ion imaging in the study of large molecule photodissociation

The Journal of Chemical Physics 147, 013904 (2017); 10.1063/1.4975671

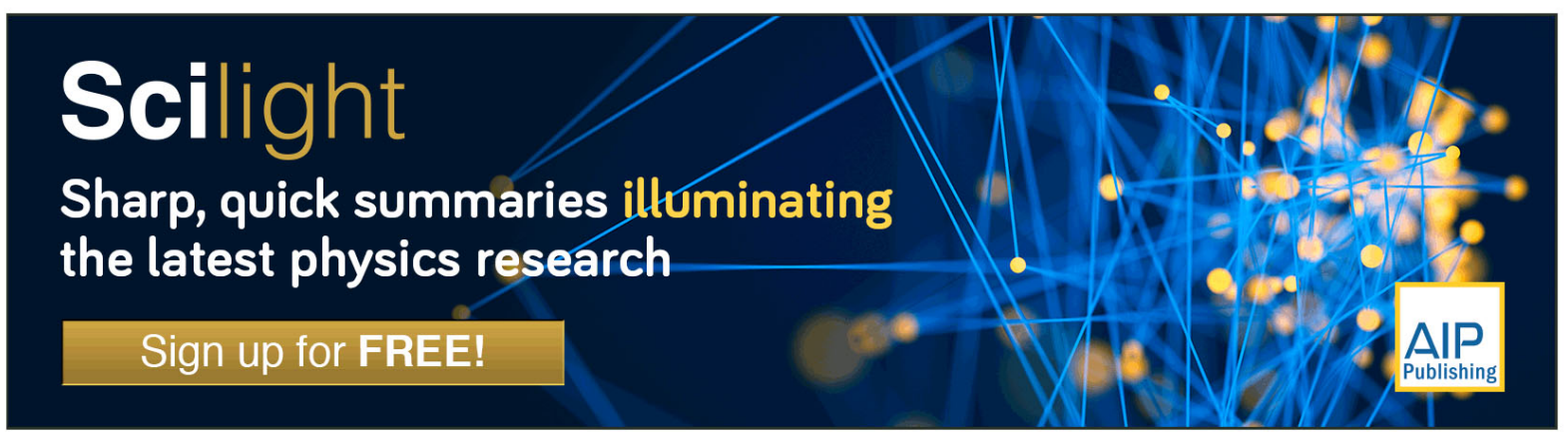




\title{
lon-ion coincidence imaging at high event rate using an in-vacuum pixel detector
}

\author{
Jingming Long (龙精明), ${ }^{1, a)}$ Federico J. Furch ${ }^{1}$, Judith Durá, ${ }^{1}$ Anton S. Tremsin, ${ }^{2}$ \\ John Vallerga ${ }^{2}$ Claus Peter Schulz, ${ }^{1}$ Arnaud Rouzée, ${ }^{1}$ and Marc J. J. Vrakking ${ }^{1, b)}$ \\ ${ }^{1}$ Max-Born-Institut für Nichtlineare Optik und Kurzzeitspektroskopie, Max-Born-Straße 2A, \\ 12489 Berlin, Germany \\ ${ }^{2}$ The Space Sciences Laboratory, University of California, Berkeley, California 94720, USA
}

(Received 31 January 2017; accepted 4 April 2017; published online 24 April 2017)

\begin{abstract}
A new ion-ion coincidence imaging spectrometer based on a pixelated complementary metal-oxidesemiconductor detector has been developed for the investigation of molecular ionization and fragmentation processes in strong laser fields. Used as a part of a velocity map imaging spectrometer, the detection system is comprised of a set of microchannel plates and a Timepix detector. A fast time-to-digital converter (TDC) is used to enhance the ion time-of-flight resolution by correlating timestamps registered separately by the Timepix detector and the TDC. In addition, sub-pixel spatial resolution $(<6 \mu \mathrm{m})$ is achieved by the use of a center-of-mass centroiding algorithm. This performance is achieved while retaining a high event rate $\left(10^{4}\right.$ per s). The spectrometer was characterized and used in a proof-of-principle experiment on strong field dissociative double ionization of carbon dioxide molecules $\left(\mathrm{CO}_{2}\right)$, using a $400 \mathrm{kHz}$ repetition rate laser system. The experimental results demonstrate that the spectrometer can detect multiple ions in coincidence, making it a valuable tool for studying the fragmentation dynamics of molecules in strong laser fields. Published by AIP Publishing. [http://dx.doi.org/10.1063/1.4981126]
\end{abstract}

\section{INTRODUCTION}

The ionization and fragmentation of molecules by intense laser fields have been the subject of intensive research during the last three decades. Over the years, several experimental techniques have been developed to record the momentum distribution of charged particles that are produced by strong field ionization. Among them, two techniques are widely used, namely, velocity map imaging (VMI) ${ }^{1,2}$ based on recording 2D projections of the momentum distributions of charged particles (electrons or ions), and 3D coincidence imaging, ${ }^{3}$ in which the 3D momenta of individual electrons and ions are measured in coincidence.

In a typical velocity map imaging (VMI) experiment, the momentum distribution of a single, positively or negatively charged particle is recorded. This is done by using a set of three electrodes that act as an electrostatic lens, in combination with a detection system. The electrostatic field accelerates the ions or electrons towards a field-free flight tube and the particles are usually detected by a microchannel plate $(\mathrm{MCP})+$ phosphor screen assembly that is observed by a charge-coupled device (CCD) or a complementary metaloxide-semiconductor (CMOS) camera. ${ }^{4-8}$ Selection of the ion mass-to-charge ratio is possible by the application of a high voltage gate on the $\mathrm{MCP}(\mathrm{s})$. An advantage of the velocity map imaging lens geometry is that the position of impact on the detector is (nearly) independent of the position of ionization and is determined almost entirely by the initial

\footnotetext{
a) Jingming.Long@mbi-berlin.de

b) Marc.Vrakking@mbi-berlin.de
}

transverse velocity of the particles (i.e., the velocity parallel to the detection plane). The measurement therefore yields the 2D momentum distribution in the plane of the detector for the mass-selected fragment. The initial 3D momentum distribution can be retrieved from this measured 2D momentum distribution using algorithms incorporating an Abel inversion procedure. It is also possible to measure slices of the $3 \mathrm{D}$ momentum distribution directly, without the need of an Abel inversion, by stretching the Newton sphere (using a special electrode design) and using the gating of the $\mathrm{MCP}(\mathrm{s})$ to restrict the detection to a subset of the ions that are produced for a particular fragment mass. ${ }^{9}$ Alternatively, gating of the camera system can be also used to achieve slice imaging. ${ }^{7,10}$ Velocity map imaging spectrometers permit experiments with large count rates and therefore are generally used in combination with low repetition rate laser sources.

Since a single velocity-map image only measures electrons or ions, and-in the latter case-typically only a single mass-to-charge fragment, it offers only a partial view of the process under investigation. More complete information can be retrieved when the momentum vectors of all fragments are measured in coincidence. Such kinematically complete experiments can be performed by using a reaction microscope $(\mathrm{REMI})^{3}$ or cold target recoil ion momentum spectrometer (COLTRIMS). ${ }^{11}$ In such a setup, positively and negatively charged particles (i.e., ions and electrons) are mapped onto two position- and time-sensitive detectors that are mounted at opposite ends of the flight tube. By applying momentum conservation, one can identify electrons and ions that emerge from the same atom or molecule in the interaction region and one can obtain the 3D momenta of all fragments. 3D coincidence 
momentum imaging (CMI) becomes exceedingly difficult if more than a few events occur per laser shot. ${ }^{12}$ Since the ratio of false to real coincidences scales very unfavourably with the number of events per laser shot, coincidence experiments favour the use of a high repetition rate laser system, so that the event rate can be kept well below one event per laser shot, without ending up with excessively long data acquisition times.

In a typical 3D coincidence experiment, the position- and time-sensitive detector is composed of two or three MCPs in combination with either a wedge-and-strip or delay-line anode. The latter is most common and in the simplest case consists of two orthogonal wound wires that are connected to two analogto-digital converters (ADCs). The particle position is obtained from the difference of the timing signals that are measured for each wire pair. Commercially available detector setups allow a spatial resolution down to $100 \mu \mathrm{m}$, whereas the particle arrival time information is extracted directly from the MCPs and can reach a sub-50 ps resolution.

Recently there have been several examples in which pixelated detectors such as CCD and CMOS cameras were used in velocity map imaging and coincidence momentum imaging. ${ }^{7,8,13,14}$ In the latter context, Lee et al. recently used a fast $128 \times 128$ pixel CMOS camera $(1 \mathrm{kHz})$ in combination with a high speed digitizer. ${ }^{15} \mathrm{~A}$ spatial resolution of $\sim 500 \mu \mathrm{m}$ and a time resolution of $\sim 200$ ps were achieved. To correlate the spatial and temporal measurements of a given particle, the amplitude signal from a single anode photomultiplier tube (PMT) was recorded by the high speed digitizer and was correlated with the particle intensity on the camera. Due to the use of a $1 \mathrm{kHz}$ laser, the data acquisition was very time-consuming. ${ }^{15}$ The use of a higher repetition rate laser could improve the situation, although the detection of multiple ions with similar intensities in the same frame introduces difficulties in the correlation of the temporal and spatial information.

Following our previous application of an in-vacuum Medipix and Timepix detector for performing VMI measurements, ${ }^{7,8}$ and the development of a high repetition rate $(400 \mathrm{kHz})$ laser system, ${ }^{16,17}$ we present a new ion velocity map imaging spectrometer based on a quad-chip (512 $\times 512$ pixels) Timepix detector and illustrate its use as an alternative to a traditional delay-line detector. The new spectrometer is characterized by detecting intense field, twobody dissociation following the double ionization of carbon dioxide $\left(\mathrm{CO}_{2}\right)$. The spectrometer combines superb spatial and temporal resolution with a $1 \mathrm{kHz}$ frame rate. Accordingly, when combined with a high repetition rate laser system, the spectrometer holds great promise for timeresolved pump-probe experiments using ion-ion coincidence imaging.

\section{EXPERIMENTAL SETUP}

The complete setup is schematically displayed in Fig. 1. Experiments are performed using a home-built $400 \mathrm{kHz}$ Optical Parametric Chirped Pulse Amplifier (OPCPA $)^{17}$ delivering $10 \mu \mathrm{J}$ few-cycle pulses $(\tau=7 \mathrm{fs})$ at $790 \mathrm{~nm}$. The output of the laser is tightly focused inside the velocity map imaging spectrometer (see Fig. 2) using a zero-degree concave mirror with $f=75 \mathrm{~mm}$ onto a continuous molecular beam obtained by expanding a gas maintained at a constant pressure ( 2 bars) through a custom-made nozzle $(\varnothing=20 \mu \mathrm{m})$. Two skimmers $(\varnothing=100 \mu \mathrm{m}$ and $\varnothing=1 \mathrm{~mm})$ located downstream of the nozzle at distances of 10 and $280 \mathrm{~mm}$ are used in order to create a cooled molecular beam and to achieve differential pumping. Under typical experimental conditions the peak laser intensity at the focus is $1.2 \times 10^{15} \mathrm{~W} / \mathrm{cm}^{2}$, and the

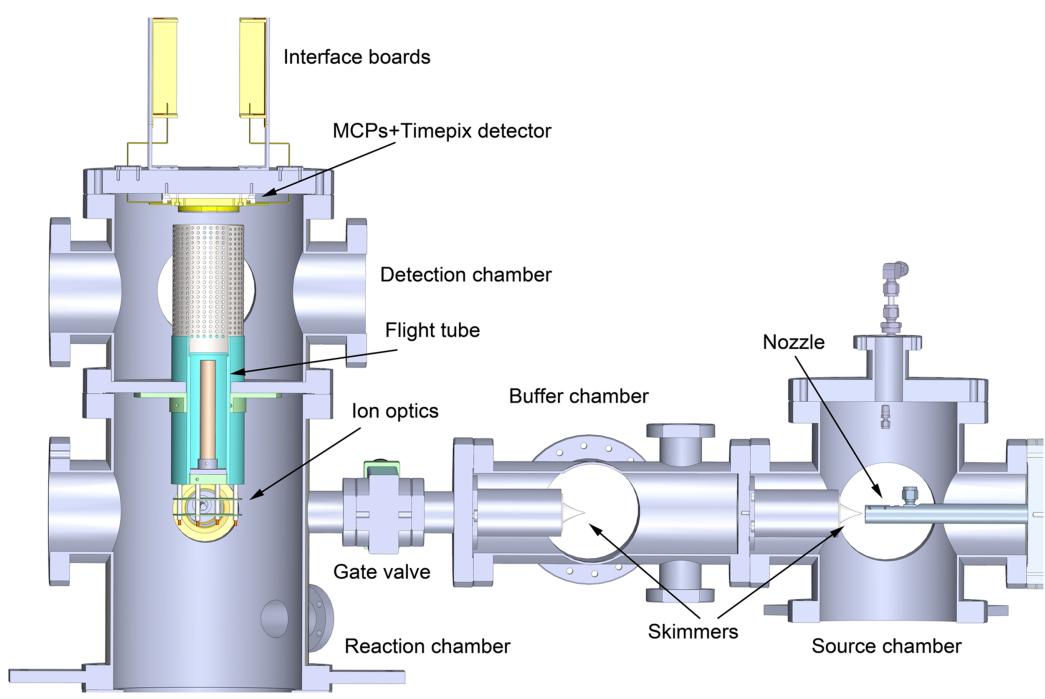

FIG. 1. Ion-ion coincidence imaging setup using an MCP + Timepix detector combination. The setup consists of two main parts that are connected by an ultra-high vacuum (UHV) gate valve. The spectrometer shown on the left includes a reaction chamber and a detection chamber, in which the electrostatic lenses and the $3 \mathrm{D}$ detector are installed, respectively. To prevent outgassing of the printed circuit board (PCB) of the pixel detector into the reaction chamber, a 14 cm long, $20 \mathrm{~mm}$ diameter pipe is mounted in the field-free TOF tube, which allows ions with a kinetic energy below $10 \mathrm{eV}$ to pass through and drift towards the detector. The MCP + Timepix detector combination is located underneath the top flange of the detection chamber and is connected by multi-pin feedthrough cables to two interface boards located outside the vacuum. The right side of the apparatus contains the molecular beam source. The sample gas exits from a custom-made nozzle located in the source chamber. After the first skimmer, a cooled supersonic molecular beam is formed and further collimated by a second skimmer located in the buffer chamber. 


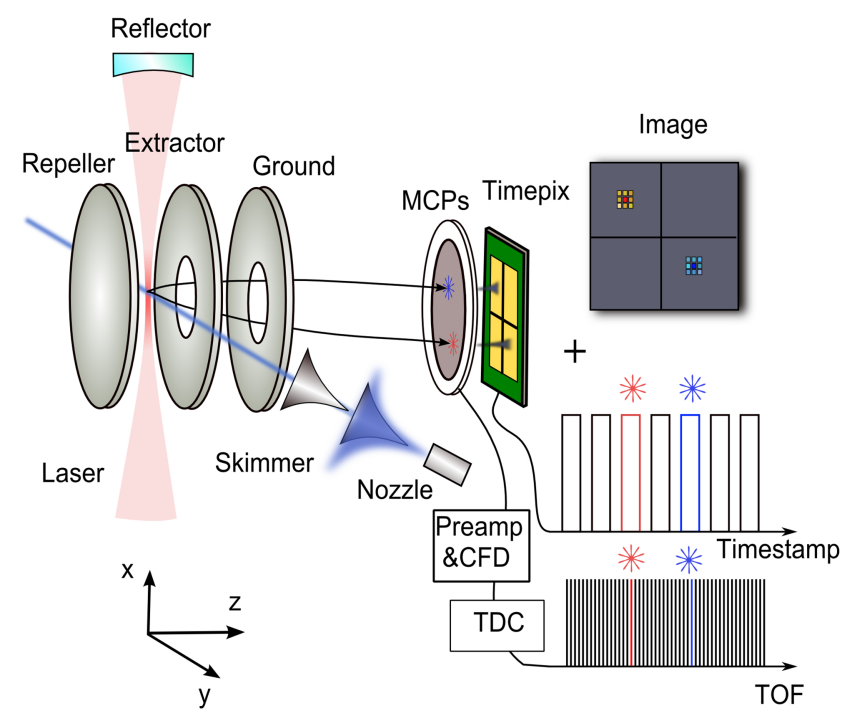

FIG. 2. Schematic diagram of the ion-ion coincidence measurement. The electrostatic lenses follow the design of Eppink and Parker ${ }^{1,2}$ and are designed and configured for velocity map imaging (VMI). The propagation direction of the laser beam (along the $\mathrm{x}$-axis) is perpendicular to the molecular beam (propagating along the y-axis), while the linear polarization of the laser beam is along the $y$-axis and thus parallel to the detection plane of the MCP + Timepix detector. The TOF axis is defined as the z-axis. Ions generated at the crossing point of the laser and the molecular beam (interaction region) are independently detected by the MCP + TDC combination and by the Timepix detector. The former provides precise TOF information limited by the $25 \mathrm{ps}$ TDC time bin, while the particle position and a rough timestamp (10 ns time bin) are recorded by the Timepix detector. Both data are finally correlated by global frame timestamps. The correlation procedure is described in more detail in the text. Preamp: preamplifier; CFD: constant fraction discriminator; TDC: time-to-digital converter.

diameter of the focal region is around $10 \mu \mathrm{m}$. The molecular density is estimated to be below $10^{8} / \mathrm{cm}^{3}$, meaning that only one or a few molecules are present in the region where the laser reaches an intensity that is high enough to ionize the molecule.

The velocity map imaging spectrometer is composed of three electrodes following the standard design described by Eppink and Parker ${ }^{1,2}$ (typically operated with $\mathrm{V}_{\text {repeller }}=16 \mathrm{kV}$, $\mathrm{V}_{\text {extractor }}=11.48 \mathrm{kV}$, and $\left.\mathrm{V}_{\text {ground }}=0 \mathrm{~V}\right)$. Charged particles produced by ionization by the few-cycle pulses are accelerated towards a 3D detector placed at the end of the $30 \mathrm{~cm}$ long field-free flight tube. The 3D detector consists of a chevronpaired MCP combination placed $1.2 \mathrm{~mm}$ in front of a Timepix detector. A mesh held at ground potential and located $5 \mathrm{~mm}$ in front of the 3D detector is used to decrease the effect of the MCP front surface voltage on the charged particle motion in the field-free drift tube. Due to the length of the free flight tube, the voltages applied and the size of the detector, charged particles up to $10 \mathrm{eV}$ kinetic energy can be detected.

The reaction chamber and the detection chamber are separated by a narrow $(\mathrm{L}=14 \mathrm{~cm}, \mathrm{D}=20 \mathrm{~mm})$ tube in order to suppress outgassing of the Timepix detector printed circuit boards (PCBs) into the reaction chamber, where the strong field ionization experiment needs to be carried out under ultrahigh vacuum (UHV) conditions. Without the injection of gas, the background pressure in the reaction chamber is $10^{-10} \mathrm{mbar}$ and the pressure in the detection chamber is $10^{-9}$ mbar.

\section{A. 3D detector}

The 3D detector is composed of a set of chevron-paired MCPs (pore size $12 \mu \mathrm{m}$, pitch size $15 \mu \mathrm{m}$, diameter $40 \mathrm{~mm}$ ) mounted in front of a quad-chip Timepix detector (see Fig. 2). A similar detector combination was previously used by our team for VMI and sliced VMI. ${ }^{7,8}$ The Timepix detector is based on CMOS technology and corresponds to the successor of the Medipix 2 detector that was developed by the Medipix collaboration coordinated by the European Organization for Nuclear Research $(\mathrm{CERN}) .{ }^{14}$ A standard Timepix chip has $256 \times 256$ pixels with a pitch of $55 \mu \mathrm{m}$ and is bump-bonded to a readout chip. Our quad-chip Timepix detector has four chips in a 2 $\times 2$ arrangement with a gap of $180 \mu$ m between adjacent chips, thus providing 262144 individual pixels on an active area of $28 \times 28 \mathrm{~mm}^{2}$. Each pixel of the detector contains a preamplifier, a discriminator with a 4-bit adjustable threshold, and a 14-bit pseudorandom counter with a counting depth of 11810 counts. Each pixel has three different operation modes ${ }^{18}$ and can be addressed independently: (1) Medipix/counting mode, where the on-board counter registers the number of times the incoming signal exceeds a specified threshold; (2) timeover-threshold (TOT) mode, where the on-board counter is continuously increased as long as the signal exceeds a specified threshold; (3) Timepix/time-of-flight (TOF) mode, where the arrival time of one impinging particle is measured with respect to an externally triggered shutter signal. After the shutter opens, the on-board counter starts running at the moment the incoming signal first exceeds a specified threshold and stops when the shutter closes. The shutter open and close signals determine the start and end times of acquisition of a frame. In the experiment, the Timepix detector is operated in the TOF mode, i.e., the impact position and the TOF of incoming particles with respect to an external trigger are recorded. The Timepix detector allows for a maximum time resolution of 10 ns (given by the $\leq 100 \mathrm{MHz}$ clock rate of the Timepix chip) and has a dynamic range up to $100 \mu \mathrm{s}$ (limited by the dynamic range of the 14-bit counter). We will see later that to achieve a better temporal resolution, the TOF data from the Timepix detector can be combined with an analog signal extracted from the MCPs and recorded using a time-to-digital converter (TDC).

\section{B. Readout system (data transfer and storage)}

The readout system for the quad-chip Timepix detector was developed at the Space Sciences Laboratory at the University of California at Berkeley ${ }^{19,20}$ and achieves a readout of $1000 \mathrm{frames} / \mathrm{s}$ using a parallel readout technique based on several field programming gate arrays (FPGAs). As shown in Fig. 1, the digital signal from each pixel of the Timepix detector is read out by two, 96-pin feedthrough cables connected to two FPGA interface boards (Xilinx Spartan 3) located outside the detection chamber and running in parallel. A larger FPGA board (Xilinx Virtex 5), the so-called Roach board, is configured in order to generate a single $512 \times 512$ image, which is then transferred to an acquisition computer using a $10 \mathrm{~Gb} / \mathrm{s}$ Ethernet interface. At $1 \mathrm{kHz}$ readout rate, the readout system has a $286 \mu \mathrm{s}$ dead time for each frame,${ }^{19-21}$ which is the minimum time required to transfer all data from the chips to the 
interface boards. After this time interval, the chips are ready to collect new events for the next frame by opening the shutter of the Timepix detector. In the TOF mode, the image data of the Timepix detector are sent to an acquisition computer by the Roach board and is processed online to generate a list of pixels that were illuminated during the opening of the shutter. Each illuminated pixel is associated with a position, specified by $x$ and $y$, and an arrival time $t$. A global time is then associated to the frame for its later identification.

\section{Time-to-digital converter and synchronization with Timepix}

When using the highest possible clock rate $(100 \mathrm{MHz})$, the Timepix detector provides TOF information with a $10 \mathrm{~ns}$ time resolution, which permits distinguishing ions that have different masses but does not give sufficiently accurate time information to draw detailed conclusions about the velocity of the ions along the electric field direction. To retrieve this velocity from the recorded TOF, a time resolution in the picosecond range is required. This is accomplished by comparing the TOF signal from the Timepix detector to the TOF signal extracted from the MCP current. Using a decoupling box, the AC signal from the rear MCP following an ion hit is extracted and preamplified, before being preprocessed by a constant fraction discriminator (CFD), and finally fed into a fast time-to-digital converter (TDC). The TDC (TDC8HP, RoentDek) has 8 NIMcompatible input channels with a 25 ps bin size (the least significant bit, LSB 25 ps) and a dead time of less than $5 \mathrm{~ns}$ for a maximum measurement range up to $419 \mu \mathrm{s}$. The TDC is controlled by the CoboldPC software from RoentDek, which also saves the experimental data in the acquisition computer for a later off-line analysis.
In the current implementation, the spectrometer is designed for working with a high repetition rate laser system $(400 \mathrm{kHz})$, and all fragments and parent ions arrive in less than $2.5 \mu$ s. In order to synchronize the TOF measurements from the MCP and the Timepix detector, a $400 \mathrm{kHz}$ photodiode signal synchronized with the pulses from the OPCPA is divided and converted to a $1 \mathrm{kHz}$ transistor-transistor logic (TTL) signal using a frequency divider. The original photodiode signal is fed into one of the TDC channels, whereas the $1 \mathrm{kHz}$ TTL signal is used to trigger the opening of the Timepix sensor shutter. The Timepix is set to record data during a $50 \mu$ s interval, and the "shutter closed" signal from the Timepix is used to trigger the TDC, thus permitting the capture with high time resolution of all ion events during the $50 \mu$ s time interval while the Timepix shutter is open (see Fig. 3(a)). In other words, during the $50 \mu$ s interval when the Timepix shutter is open, ions generated by the 20 laser pulses that occur during this interval are both recorded by the Timepix detector (and turned into an $(x$, $y, t$ ) entry in the Timepix data list) and, separately, by the TDC. Both the frame from the Timepix detector and the TDC data are assigned a global timestamp. Based on these timestamps, one can correlate the position and the (rough) time information from the Timepix detector with precise TOF data recorded from the TDC.

In Fig. 3(b), a typical distribution of the number of correlated events per frame is shown as well as the distribution of events recorded by the TDC and Timepix detector. In our chosen experimental conditions, we observed an average of 9-10 events/frame with the Timepix detector (i.e., about 0.5 event/laser shot), whereas an average of 6-7 events/frame is detected with the TDC. The lower detection efficiency of the TDC is due to the relatively high threshold used in the (a)

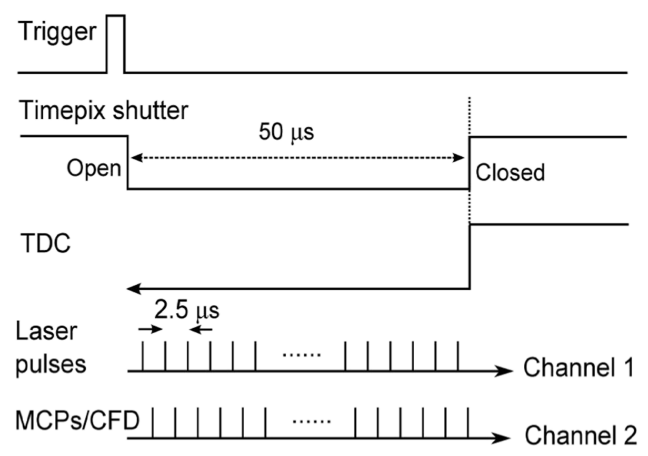

(b)
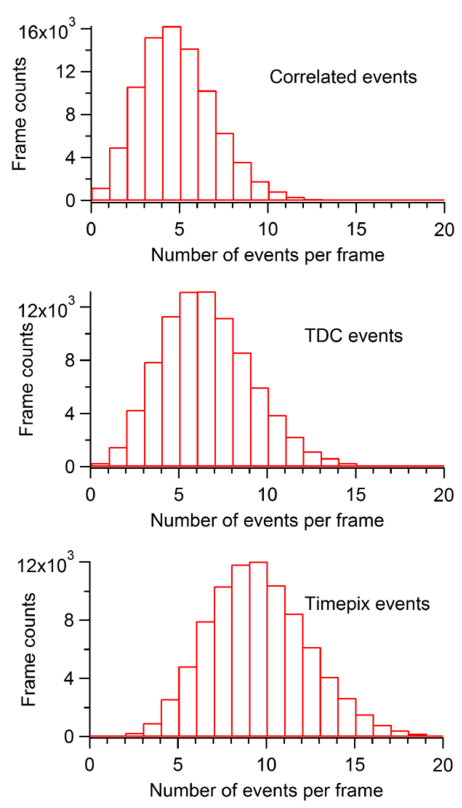

FIG. 3. (a) Timing diagram of the data acquisition. The Timepix detector is triggered by $1 \mathrm{kHz}$ TTL pulses from a frequency divider that divides a diode signal derived from the laser $(400 \mathrm{kHz})$ to the maximum frame readout rate of the Timepix $(1 \mathrm{kHz})$. A typical measurement time window is $50 \mu \mathrm{s}$, during which 20 laser pulses are fired onto the molecular beam. The TDC is triggered by the "shutter closed" edge of the Timepix detector and registers all events that occur on its channels relative to this edge. The laser signal from the diode and the pick-off signal from the MCPs are fed into two of the TDC channels in order to retrieve the TOF. (b) Distribution of the number of events per frame in our test experiment. Top: distribution of correlated (i.e., Timepix + TDC) events. Middle: distribution of events measured with the TDC. Bottom: distribution of events measured by the Timepix detector. The ratio of the number of correlated events to the number of TDC and Timepix events allows us to determine that the efficiency of the Timepix detection is $86 \%$ and that of the TDC detection is $57 \%$ (see text for details). 
constant fraction discriminator (CFD) in order to suppress false events caused by the ringing of the MCP pick-up circuit, thereby however filtering out some true events where the MCP gain is small. While such events may remain below the CFD threshold, they are nevertheless likely to be recorded by the Timepix detector. We note as well that due to the $5 \mathrm{~ns}$ dead time of the TDC, pairs of ions with a very similar TOF, for instance, two atomic $\mathrm{O}^{+}$ions that are ejected parallel to the detection plane, are registered as a single particle by the TDC. However, the position of both ions is still recorded in the Timepix image and is distinguishable. Overall, we can conclude from the number of correlated events, the number of Timepix-only events, and the number of TDC-only events that an average detection efficiency of $86 \%$ is achieved with the Timepix detector, whereas the average detection efficiency is about $57 \%$ for the TDC. N.B. These numbers do not depend on the MCP detection efficiency but describe the probability that an ion signal that is amplified by the MCPs will be detected by the Timepix and the TDC, respectively.

\section{METHOD}

\section{A. Centroiding}

Information about the ion momentum distribution in the detector plane can be directly obtained from the position of the pixels that record an ion hit. However, an individual ion event will be recorded on a number of adjacent pixels, mainly due to the spreading of the electron cloud from the MCPs that is projected onto the Timepix detector. The number of illuminated pixels is governed by the MCP gain, which depends on the voltage applied to the MCP, as well as on the voltage between the rear MCP and the Timepix chip. ${ }^{7,8}$ Saturation of the Timepix detection efficiency was observed when a voltage of $1.7 \mathrm{kV}$ was applied across the MCPs $(-2.7 \mathrm{kV}$ on the front face and $-1 \mathrm{kV}$ at the back), and using a voltage of $1.0 \mathrm{kV}$ between the rear MCP and the Timepix detector. Under these conditions, a cluster size of around $8 \times 8$ pixels was observed for each detected ion.

As previously described, the counter of each pixel begins to count in the TOF mode as soon as the input charge exceeds a preset threshold and continues until the shutter closes. Within a cluster, a larger number of electrons from the MCPs will tend to hit the central pixels than the surrounding ones; therefore, the central pixels go over the threshold earlier and register earlier TOFs than the surrounding pixels. So the recorded TOFs of pixels in a cluster reflect the spatial distribution of the electron cloud that impacts on the Timepix detector, which can be exploited in an off-line centroiding analysis. The center of the ion hit can thus be determined with sub-pixel spatial resolution. ${ }^{7,22}$

The equations for center-of-mass centroiding for a periodic pixel detector with pixel coordinates $\left(x_{i}, y_{i}\right)$ and TOFs $T_{i}$ are given as

$$
x_{c}=\frac{\sum_{i=1}^{N} x_{i} \Delta T_{i}}{\sum_{i=1}^{N} \Delta T_{i}},
$$

$$
y_{c}=\frac{\sum_{i=1}^{N} y_{i} \Delta T_{i}}{\sum_{i=1}^{N} \Delta T_{i}},
$$

where $\Delta T_{i}$ is the time difference between the arrival time $T_{i}$ recorded at the $i$ th pixel and the pixel with the earliest arrival time in the cluster. $N$ is the total number of pixels where the ion count has led to a response. $\left(x_{c}, y_{c}\right)$ represents the centroid of the detected ion event.

To determinate the spatial resolution of the MCP + Timepix detector, a home-made mask was placed $5 \mathrm{~mm}$ in front of the MCP + Timepix detector. Five groups of lines with widths and gaps between the lines ranging from 20 to $60 \mu \mathrm{m}$ were fabricated by laser machining. The transmission through the mask was recorded on the Timepix detector and the centroiding procedure described above was used. The result is shown in Fig. 4(a). Remarkably, the transmitted distribution contains a pattern of dots, indicating that the spatial resolution resulting from the centroiding procedure allows one to recognize from which channels of the MCP the electron cloud emerged.

Signal intensity distributions as a function of the horizontal image coordinate, i.e., $F(x)$, were obtained by integrating the measured images over the vertical $(y)$ coordinate. The final resolution of the detector was then estimated by fitting the resulting functions to the following equations: ${ }^{23}$

$$
\begin{gathered}
F(x)=M\left(2+\operatorname{Erf}\left(\frac{x-x_{1}}{\sqrt{2} \sigma}\right)-\operatorname{Erf}\left(\frac{x-x_{2}}{\sqrt{2} \sigma}\right)\right)+C, \\
\operatorname{Erf}(x)=\frac{2}{\pi} \int_{0}^{x} \exp \left(-t^{2}\right) d t,
\end{gathered}
$$

where $M$ is a normalization factor, $x_{1}$ and $x_{2}$ are the positions of the rising and falling edge in the transmitted pattern, and $\sigma$ quantifies the spatial resolution. In this expression, the same amplitude is assumed to be constant between the rising and falling edges. The result of the fitting procedure is given in Fig. 4(b) for the $60 \mu \mathrm{m}$ set of lines. A spatial resolution of $\sigma=5.7 \pm 0.3 \mu \mathrm{m}$ is determined in this manner, representing an upper limit to the actual spatial resolution, given the finite quality of the laser-machined masks. As noted previously, after centroiding the MCP pores can be clearly resolved in Fig. 4(a). The integrated signal, $F(x)$, along a line connecting the individual pores of the MCP pattern is given in Fig. 4(c). From this curve the pitch of the MCP can be estimated to be $14.6 \mu \mathrm{m}$, in good agreement with the specification of the MCPs.

We note that there exists a trade-off between the ion detection efficiency on the Timepix detector and the ability to apply center-of-mass centroiding on the one hand, and the maximum number of ion events that can be recorded in one frame (i.e., within $1 \mathrm{~ms}$ ) without overlap between different ion counts on the other hand. Both aspects depend on the electron cloud size on the detector. Therefore, a better spatial resolution than the resolution quoted above may be achievable at the expense of the permissible count rate. 
(a)

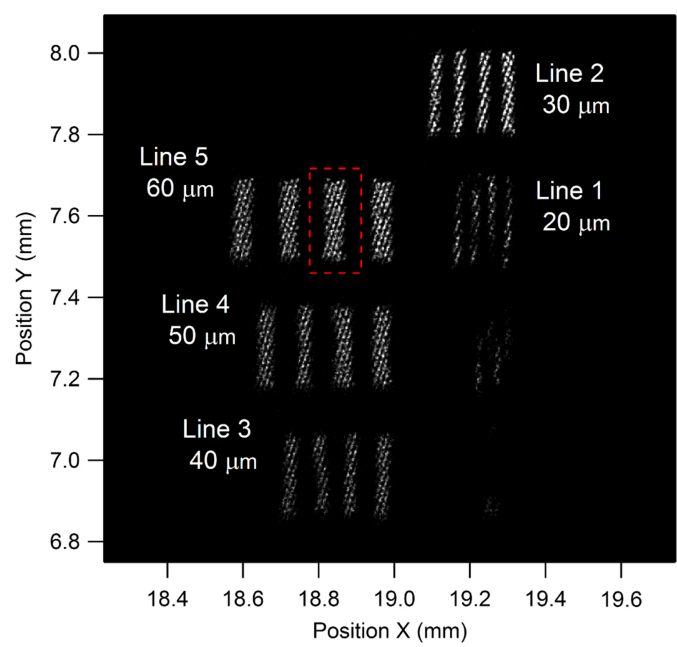

(b)
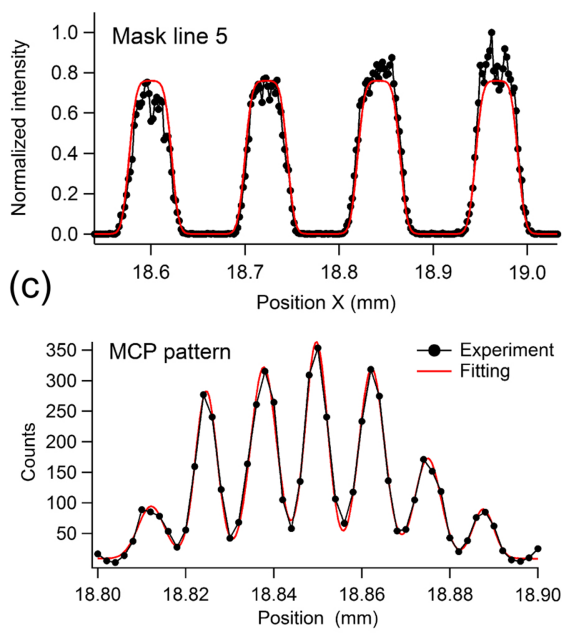

FIG. 4. (a) 2D image of ions transmitted through a resolution mask placed $5 \mathrm{~mm}$ before the MCP + Timepix detector, after centroiding. The mask contains five different groups of lines characterized by different slit widths produced by laser machining. The slits are separated by an un-machined area that has a width equal the slit width. (b) Integrated intensity distributions for the mask line 5. The data are acquired in the TOF mode of the Timepix detector. Fitting of the ion transmission through all slits suggests that the spatial resolution is $\sigma=5.7 \pm 0.3 \mu \mathrm{m}$. (c) Spatial distribution obtained by integrating the intensity within the red rectangle in Fig. 4(a) along the pitch direction. The resulting distribution was fitted by a superposition of Gaussian functions and the separation between these Gaussian functions was used to determine the pitch of the MCPs.

\section{B. Determination of 3D momentum distribution}

Once the precise spatial coordinates and the TOF of a specific ion have been measured, the 3D momentum can be calculated. In accordance with Fig. 2, we define the laser propagation direction as the $\mathrm{x}$-axis, the opposite direction of the molecular beam propagation as the $y$-axis, and the TOF axis as the $\mathrm{z}$-axis. The $\mathrm{x}$ - and $\mathrm{y}$-components of the initial 3D momentum, $p=\left(p_{x}, p_{y}, p_{z}\right)$, of an individual ion can be retrieved from its recorded displacement from the center of the detector plane $(\Delta x, \Delta y)$ and its TOF $T$, according to ${ }^{24}$

$$
\begin{aligned}
& P_{x}=\alpha \frac{m \Delta x}{T}, \\
& P_{y}=\alpha \frac{m \Delta y}{T},
\end{aligned}
$$

whereas the momentum component along the electric field $p_{z}$ is given by

$$
P_{z}=\beta \frac{\left(U_{r}-U_{e}\right) q}{d}\left(T_{0}-T\right) .
$$

In these equations $m$ and $q$ are the mass and the charge of the ion, $\Delta x$ and $\Delta y$ are the displacements of the ion from the center of the detector (i.e., from the location on the detector where an ion with $p_{x}=p_{y}=0$ will hit), $T$ is the ion time-of-flight, $T_{0}$ is the time-of-flight if $p_{z}$ is equal to zero, $d$ is the distance between the repeller and extractor electrodes, and $U_{r}$ and $U_{e}$ are the voltages applied to the repeller and accelerator plate electrodes, respectively. The coefficients $\alpha$ and $\beta$ account for the effects of the finite time interval during which the ions are accelerated, as well as the magnification effect resulting from the inhomogeneity of the electric field in the extraction region. The coefficient $\alpha$ has been calibrated as $0.78 \pm 0.03$ by fitting the electron spectrum recorded following above threshold ionization (ATI) of krypton atoms. To calibrate the coefficient $\beta, \mathrm{O}^{+}$ions were measured that resulted from the dissociative double ionization of $\mathrm{CO}_{2}$, exploiting the fact that, due to cylindrical symmetry, the momentum distributions in the $(x, y)$ plane and in the $(y, z)$ plane are identical. The $\beta$ value was determined as $1.12 \pm 0.05$ and was confirmed by trajectory simulations using the SIMION program (version 8.0). ${ }^{25}$

\section{PROOF-OF-PRINCIPLE EXPERIMENT}

As a proof-of-principle experiment, dissociative double ionization of carbon dioxide $\left(\mathrm{CO}_{2}\right)$ by an intense femtosecond laser field was investigated. For this experiment, the laser field polarization was set parallel to the detection plane. All the other experimental parameters were identical to those described in Section II. Typical position and arrival time information recorded by the Timepix detector are shown in Fig. 5. The 2D image in Fig. 5(a) is a projection of all ions produced in the experiment after applying the center-of-mass centroiding described previously. In the image, both the mesh at the end of the field-free flight tube with $34 \mu \mathrm{m}$ wire diameter and the $180 \mu \mathrm{m}$ gap between the adjacent Timepix chips are clearly seen, in agreement with the previously determined sub- $6 \mu \mathrm{m}$ spatial resolution. As expected, the TOF histogram displayed in Fig. 5(b) shows that the $50 \mu$ s measurement time window contains 20 laser pulses separated by a $2.5 \mu$ s interval. The assignment of the ion peaks in the TOF spectra is shown in the inset of Fig. 5(b). The extensive production of $\mathrm{CO}_{2}{ }^{+}$ parent molecular ions results in a pronounced detection loss in the central part of the detector, as indicated by the green dashed line in Fig. 5(b), which shows that after the first few laser pulses and, correspondingly, detection of the first $\mathrm{CO}_{2}{ }^{+}$ parent ion, the detection of further $\mathrm{CO}_{2}{ }^{+}$within the same camera frame is compromised. The reason is that in the TOF mode, each pixel of the Timepix detector can register at most one event in a single frame $(1 \mathrm{~ms})$. Therefore, all ion events following the first hit of a given pixel are discarded during 
(a)

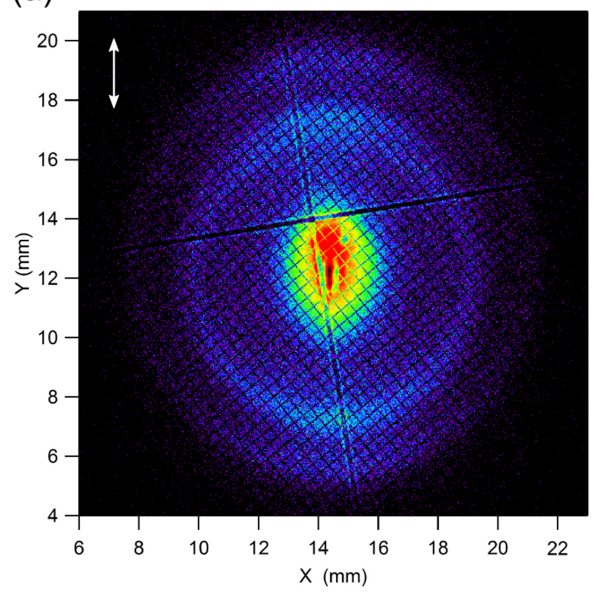

(b)

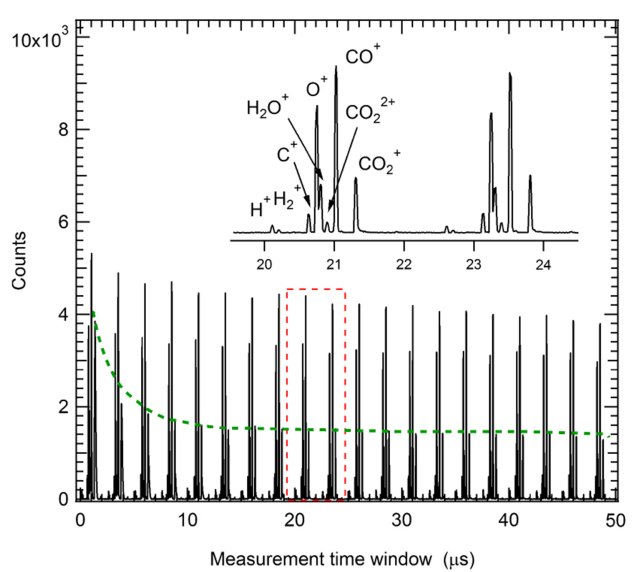

FIG. 5. (a) Raw 2D spatial image after centroiding representing the projection of all ions produced in the proof-of-principle experiment on the strong field (dissociative) ionization of $\mathrm{CO}_{2}$. The mesh in front of the $3 \mathrm{D}$ detector that serves to decrease the effect of the MCP voltages on the ion trajectories in the field-free drift tube is clearly visible, as are the $180 \mu \mathrm{m}$ gaps between the individual Timepix chips. The white double-sided arrow indicates the direction of the laser polarization. The Timepix chips are rotated by $9^{\circ}$ with respect to the laser polarization. (b) TOF distributions obtained directly from the Timepix detector, after accumulating $1 \times 10^{6}$ frames. There are 20 laser pulses within each frame, so that the TOF spectrum is replicated 20 times. Two of the TOF distributions are shown in the inset. The peaks are assigned to specific ions. A green dashed line is plotted to draw attention to the intensity decrease of the parent ion $\mathrm{CO}_{2}{ }^{+}$signal as a function of the measurement time within the frame. This is due to pile-up of parent ions on the central part of the detector.

the shutter opening time (50 $\mu \mathrm{s})$. In contrast, the peak intensities of the $\mathrm{O}^{+}$and $\mathrm{CO}^{+}$ion peak decrease only slightly. This is a direct consequence of the fact that these fragment ions have a finite kinetic energy release. Therefore there is a low probability that within one frame ions will land multiple times at the same location on the detector. ${ }^{26}$ One can estimate that the probability of overlapping electron clouds created by different fragment ions within one frame increases to $100 \%$ when the ionization rate is higher than 3 events/laser shot. In what follows, we disregard the parent ion detection in the central part of the Timepix detector, which anyway does not contribute to coincident events.

In the experiment, the ionization rate depends on the laser intensity, the focal volume, and the density of the molecular beam in the interaction region. In the experiment shown in Fig. 5, the ion event rate is around 0.5 counts/laser shot. We note that a rate of 0.5 events/laser shot is near the upper limit that one can afford in a coincidence experiment to avoid false coincidences. Combining the high repetition rate laser system and the fast MCP + Timepix detector, a usable total ion event rate of $10^{4}$ per $\mathrm{s}$ is available in the ion-ion coincidence measurements.

Compared to the rough timing signal of the fragment ions obtained from the Timepix, a TOF spectrum with higher time resolution (limited only by the arrival time distribution of the ions and the $25 \mathrm{ps}$ bin time of the TDC) is obtained from the TDC acquisition. This can potentially enhance the TOF time resolution about 400 -fold. Indeed, in a mass spectrum recorded for Xe (not shown), the mass resolution of the spectrometer was determined to be better than $\mathrm{M} / \Delta \mathrm{M}=2700$ and the width of individual mass peaks was below $1 \mathrm{~ns}$. A mass spectrum acquired using the TDC data is shown on both a linear and a logarithmic scale in Fig. 6. Fragments resulting from dissociative ionization and Coulomb explosion of $\mathrm{CO}_{2}$, including dominant contributions from $\mathrm{C}^{+}, \mathrm{O}^{+}$, and $\mathrm{CO}^{+}$and smaller amounts of $\mathrm{C}^{2+}$ and $\mathrm{O}^{2+}$ ions, can be readily observed.
Due to the very high mass resolution, different isotopes are clearly separated in the mass spectrum. Among the many ions in the mass spectrum, a contribution corresponding to the detection of the metastable $\mathrm{CO}_{2}{ }^{2+}$ ion is also observed. In what follows, two-body dissociation of the $\mathrm{CO}_{2}{ }^{2+}$ ion will be discussed to demonstrate the utility of our spectrometer for ion-ion coincidence imaging.

\section{A. Two-body dissociation of $\mathrm{CO}_{2}{ }^{2+}$}

Dissociative double ionization of $\mathrm{CO}_{2}$ has been studied by using electron impact, synchrotron light, and intense femtosecond laser pulses. ${ }^{26-29}$ Typical dissociation channels of $\mathrm{CO}_{2}{ }^{2+}$ are (i) $\mathrm{C}^{+}+\mathrm{O}^{+}+\mathrm{O}$, (ii) $\mathrm{C}+\mathrm{O}^{+}+\mathrm{O}^{+}$, and (iii) $\mathrm{CO}^{+}$ $+\mathrm{O}^{+}$. Here, we will focus in particular on the latter channel, i.e., the production of $\mathrm{CO}^{+}+\mathrm{O}^{+}$. There are two mechanisms

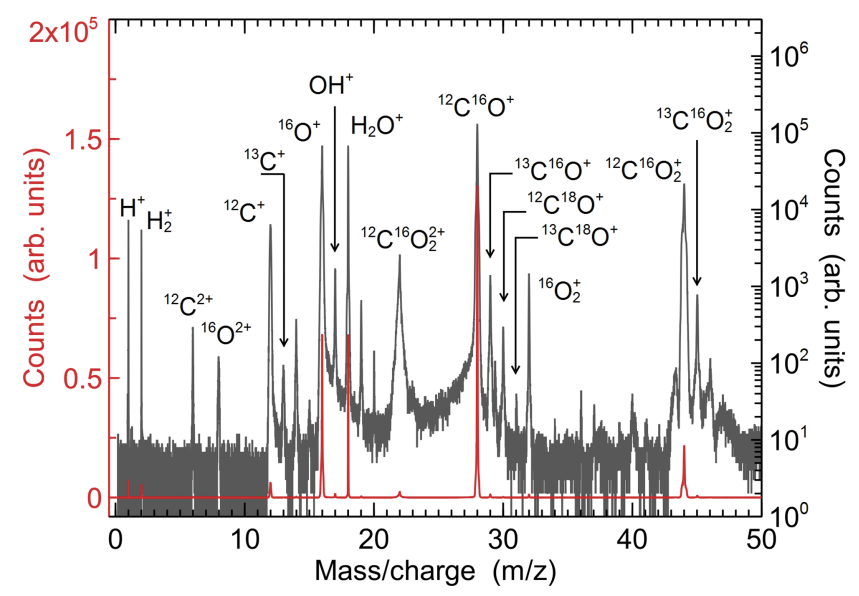

FIG. 6. High resolution mass spectrum for the ionization of $\mathrm{CO}_{2}$ in a strong laser field, obtained using the timing information from the TDC, for the laser conditions given in Section IV. The left axis is plotted on a linear scale and refers to the curve plotted in red, while the right axis is plotted on a logarithmic scale and refers to the curve plotted in black, which permits the observation of weaker signals. 
(a)

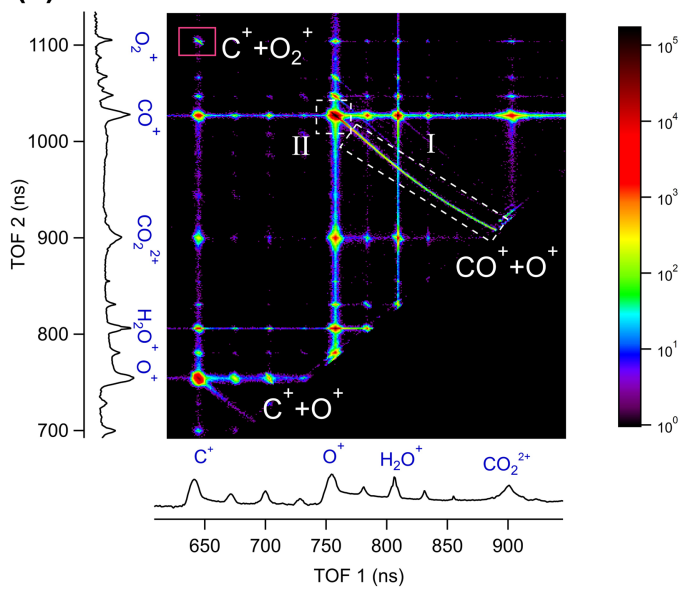

(b)

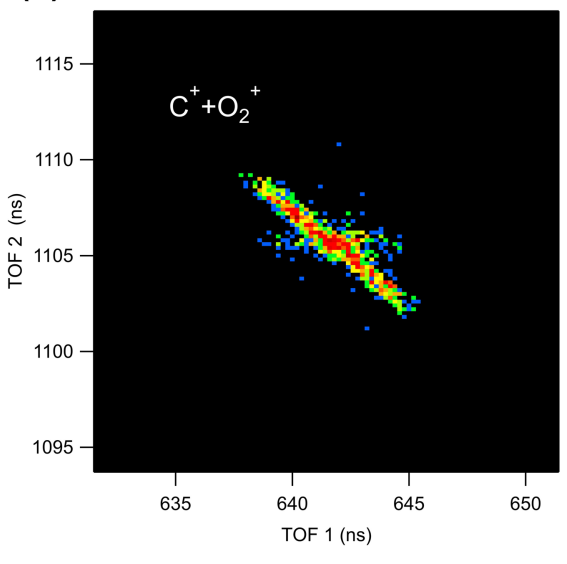

FIG. 7. (a) Ion-ion coincidence image on the basis of TOF data retrieved from the TDC. As elaborated in the text, the formation of the ion pair $\left(\mathrm{CO}^{+}, \mathrm{O}^{+}\right)$ occurs by two different fragmentation mechanisms (leading to regions I and II). The ion-pair $\left(\mathrm{C}^{+}, \mathrm{O}_{2}{ }^{+}\right)$is observed within the red rectangle and shown on a larger scale in (b). The anti-correlation between $\mathrm{C}^{+}$and $\mathrm{O}_{2}{ }^{+}$flight-times is a result of momentum matching of the two fragments in the break-up of $\mathrm{CO}_{2}{ }^{2+}$. for the formation of this channel, accompanied by different time scales for the production of the ion-pair. ${ }^{26,28}$ One contribution is the dissociation of the metastable parent ion $\mathrm{CO}_{2}{ }^{2+}$ that occurs with a lifetime of approximately $3.1 \mu \mathrm{s} .{ }^{28}$ In our experiments, a part of this dissociation happens during the extraction of the ions in the velocity map imaging spectrometer. This can be seen in the mass spectrum shown in Fig. 6, where the $\mathrm{O}^{+}$and $\mathrm{CO}^{+}$flight-times are not at all sharp and sit on the top of long pedestals that extend towards the flight time of $\mathrm{CO}_{2}{ }^{2+}$. The reason for this behavior becomes more transparent when plotting an ion-ion coincidence map as shown in Fig. 7(a). In this plot the correlation is shown between the times-of-flight of any two ions that together form a coincident ion pair. Coincident $\mathrm{O}^{+}$and $\mathrm{CO}^{+}$ions that are formed shortly after the laser excitation are observed at the timesof-flight expected for $\mathrm{m} / \mathrm{z}=16$ (TOF $=\sim 760 \mathrm{~ns}$ ) and $\mathrm{m} / \mathrm{z}=28$ (TOF $=\sim 1030 \mathrm{~ns})$, whereas $\mathrm{O}^{+}$and $\mathrm{CO}^{+}$ions that are formed when the acceleration in the extraction region has been completed are measured near the time-of-flight of parent $\mathrm{CO}_{2}{ }^{2+}$ ions $(\mathrm{m} / \mathrm{z}=22, \mathrm{TOF}=\sim 900 \mathrm{~ns}) . \mathrm{O}^{+}$and $\mathrm{CO}^{+}$ions that are formed in between these two times are detected on the line (labeled as region I in Fig. 7(a)) connecting the point corresponding to prompt coincident $\mathrm{O}^{+}+\mathrm{CO}^{+}$detection and the point in the coincidence map where both ion 1 and ion 2 have the time-of-flight of $\mathrm{CO}_{2}{ }^{2+}$. We note that since these ions are formed from a metastable $\mathrm{CO}_{2}{ }^{2+}$ ion that has a lifetime that is significantly longer than the rotational period of $42.7 \mathrm{ps}$ of $\mathrm{CO}_{2}$, these $\mathrm{O}^{+}$and $\mathrm{CO}^{+}$fragment ions have a nearly isotropic angular distribution.

The region II in Fig. 7(a) shows a significant enhancement in the $\mathrm{O}^{+}+\mathrm{CO}^{+}$contribution near the expected flight times for $\mathrm{O}^{+}$and $\mathrm{CO}^{+}$. This shows that in addition to the slow fragmentation process discussed above, there also exists an "ultrafast" fragmentation process according to a different dissociation mechanism, e.g., a prompt Coulomb explosion. Indeed, when the 2D momentum distribution (in the plane of the detector) of $\mathrm{O}^{+}$and $\mathrm{CO}^{+}$ions is plotted that result from this ultrafast fragmentation process (see Fig. 8), a pronounced anisotropy is observed in the angular distribution, where the $\mathrm{O}^{+}$and $\mathrm{CO}^{+}$angular distributions peak along the laser polarization axis. Previously, the "ultrafast" dissociation of $\mathrm{CO}_{2}{ }^{2+}$ was estimated to happen on femtosecond time scales, i.e., on a time scale much shorter than the rotational period. ${ }^{26}$ The total kinetic energy release for the prompt $\mathrm{O}^{+}$and $\mathrm{CO}^{+}$channels shown in Fig. 9 exhibits a $2 \mathrm{eV}$ broad peak centered around $5.5 \mathrm{eV}$ and agrees with previous work. ${ }^{26,30}$

In addition to the two $\mathrm{O}^{+}+\mathrm{CO}^{+}$pathways, the ion-ion coincidence map reveals a number of additional pathways. (a)

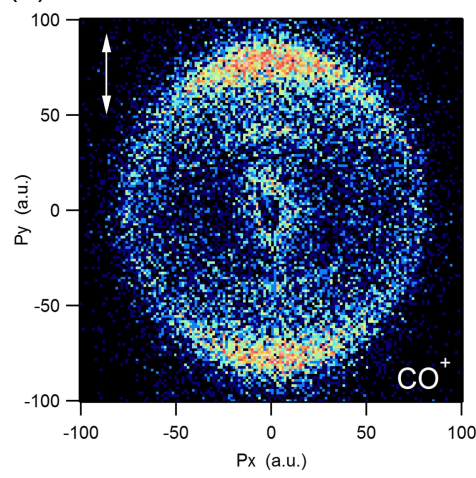

(b)

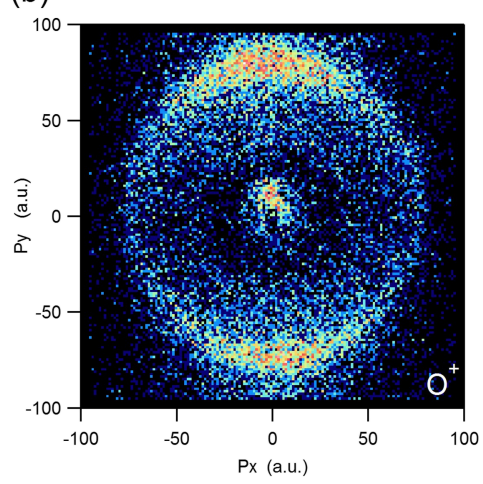

(c)

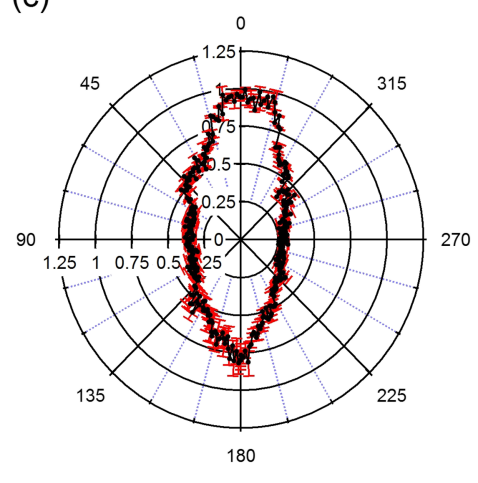

FIG. 8. Momentum distributions of $\mathrm{CO}^{+}$(a) and $\mathrm{O}^{+}$ions (b) in the break-up of $\mathrm{CO}_{2}{ }^{2+}$ into $\mathrm{CO}^{+}+\mathrm{O}^{+}$. The $\mathrm{CO}^{+}$and $\mathrm{O}^{+}$ion events are extracted from region II in Fig. 7(a). The white double-arrow line indicates the laser polarization direction. (c) Normalized angular distribution for the channel $\left(\mathrm{CO}^{+}, \mathrm{O}^{+}\right)$in region II. The angle that is plotted gives the direction of the $\mathrm{O}^{+}$ejection with respect to the laser polarization direction. N.B. The angular distribution shows a modest up-down asymmetry that is not caused by an asymmetry in the dissociative ionization process, but which is likely due to a slight misalignment of the time-of-flight tube at the time that the experiments were performed. 


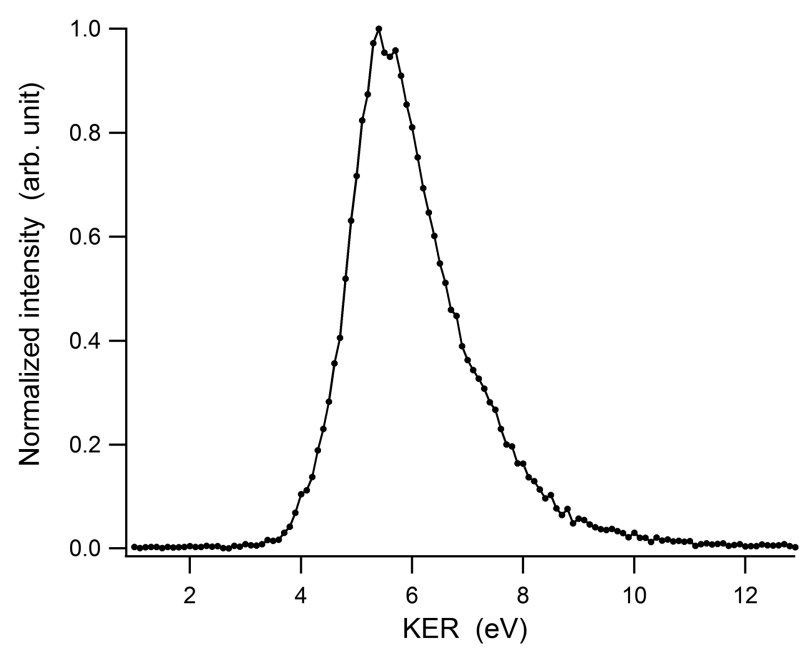

FIG. 9. Normalized kinetic energy release (KER) distribution for the channel $\left(\mathrm{CO}^{+}, \mathrm{O}^{+}\right)$.

One of these is the coincident detection of $\mathrm{C}^{+}(\mathrm{m} / \mathrm{z}=12$, $\mathrm{TOF}=\sim 640 \mathrm{~ns}$ ) and $\mathrm{O}^{+}$, pointing at the participation of the afore-mentioned $\mathrm{C}^{+}+\mathrm{O}^{+}+\mathrm{O}$ and $\mathrm{C}+\mathrm{O}^{+}+\mathrm{O}^{+}$pathways. Other contributions result from false coincidences, e.g., coincidences between the detection of $\mathrm{H}_{2} \mathrm{O}^{+}(\mathrm{m} / \mathrm{z}=18, \mathrm{TOF}=\sim 810 \mathrm{~ns})$ and $\mathrm{C}^{+} / \mathrm{O}^{+}$, and coincidences between the detection of $\mathrm{CO}_{2}{ }^{2+}$ $(\mathrm{m} / \mathrm{z}=22$, TOF $=\sim 900 \mathrm{~ns})$ and $\mathrm{C}^{+} / \mathrm{O}^{+} / \mathrm{H}_{2} \mathrm{O}^{+}$. These false coincidences, which clearly show up in the current measurements because of the very high dynamic range of the experiment (spanning about 5 orders of magnitude), underscore the importance of performing coincidence experiments at very low event rates ( $\ll 1$ event/laser shot). In addition to these false coincidences, however, Fig. 7(a) also clearly reveals a further fragmentation pathway for the $\mathrm{CO}_{2}$ molecule, namely, the formation of $\mathrm{C}^{+}$and $\mathrm{O}_{2}{ }^{+}$ion-pairs. This contribution is shown within the red rectangle in Fig. 7(a) and replotted on a larger scale in Fig. 7(b). The same finding was recently reported by Larimian et al. ${ }^{30}$ on the basis of COLTRIMS measurements. However, in contrast with the results presented there, our data do not show any evidence for a slow dissociation pathway into $\mathrm{C}^{+}+\mathrm{O}_{2}{ }^{+}$ion analogous to the previously discussed dissociation into $\mathrm{O}^{+}+\mathrm{CO}^{+}$(see Figs. 7(a) and 7(b)), even though the dynamic range in our measurement exceeds the dynamic range in the results presented by Larimian by at least one order of magnitude. The ion-ion coincidence map shown in Fig. 7(a) suggests that in our experiment the fragmentation of $\mathrm{CO}_{2}{ }^{2+}$ into $\mathrm{C}^{+}+\mathrm{O}_{2}{ }^{+}$occurs on an "ultrafast" time scale and suggests that the fragmentation of the metastable $\mathrm{CO}_{2}{ }^{2+}$ ion does not contribute to $\mathrm{O}_{2}{ }^{+}$ion formation. To investigate the fragmentation process leading to the formation of $\mathrm{C}^{+}+\mathrm{O}_{2}{ }^{+}$further, and to resolve the discrepancy between our and Larimian's result, a time-resolved ion-ion coincidence imaging experiment using a pump-probe technique is needed. This will be the subject of future work.

\section{CONCLUSION}

A new compact ion-ion coincidence spectrometer has been developed for strong field ionization and fragmentation studies. The spectrometer uses the combination of an MCP
+ Timepix detector in a UHV detection chamber. The time resolution of the Timepix detector is significantly improved by correlating the timestamp provided by the Timepix detector with a precise timestamp obtained from a fast TDC that is fed with a capacitively decoupled MCP signal. In addition, center-of-mass centroiding significantly improves the spatial resolution of the detector. To illustrate the utility of the detector, a high repetition rate laser system was employed to dissociatively ionize the $\mathrm{CO}_{2}$ molecule. An ion-ion coincidence map revealed the evidence of both a fast and a slow pathway for the dissociation of $\mathrm{CO}_{2}{ }^{2+}$ into $\mathrm{CO}^{+}$and $\mathrm{O}^{+}$. In addition, the prompt formation of $\mathrm{C}^{+}$and $\mathrm{O}_{2}{ }^{+}$ion-pairs was observed. The new spectrometer paves the way to performing time-resolved pump-probe experiments with coincident ion-ion detection in the near future.

\section{ACKNOWLEDGMENTS}

This work was financially supported by the EU-project FLAME Project (No. FP7-SME-2012-315744). J.L. would like to thank Julia Jungmann, Jan Visser, and Joop Rovekamp for their help with the Timepix detector and Achut Giree for his assistance in preparing the high repetition rate laser system.

${ }^{1}$ A. T. J. B. Eppink and D. H. Parker, Rev. Sci. Instrum. 68, 3477 (1997).

${ }^{2}$ D. H. Parker and A. T. J. B. Eppink, J. Chem. Phys. 107, 2357 (1997).

${ }^{3}$ J. Ullrich, R. Moshammer, A. Dorn, R. Dörner, L. P. H. Schmidt, and H. Schmidt-Böcking, Rep. Prog. Phys. 66, 1463 (2003).

${ }^{4}$ M. Brouard, E. K. Campbell, A. J. Johnsen, C. Vallance, W. H. Yuen, and A. Nomerotski, Rev. Sci. Instrum. 79, 123115 (2008).

${ }^{5}$ M. Brouard, E. Halford, A. Lauer, C. S. Slater, B. Winter, W. H. Yuen, J. J. John, L. Hill, A. Nomerotski, A. Clark, J. Crooks, I. Sedgwick, R. Turchetta, J. W. Lee, C. Vallance, and E. Wilman, Rev. Sci. Instrum. 83, 114101 (2012).

${ }^{6}$ A. T. Clark, J. P. Crooks, I. Sedgwick, R. Turchetta, J. W. L. Lee, J. J. John, E. S. Wilman, L. Hill, E. Halford, C. S. Slater, B. Winter, W. H. Yuen, S. H. Gardiner, M. L. Lipciuc, M. Brouard, A. Nomerotski, and C. Vallance, J. Phys. Chem. A 116, 10897 (2012).

${ }^{7}$ J. H. Jungmann, A. Gijsbertsen, J. Visser, J. Visschers, R. M. A. Heeren, and M. J. J. Vrakking, Rev. Sci. Instrum. 81, 103112 (2010).

${ }^{8}$ G. Gademann, Y. Huismans, A. Gijsbertsen, J. Jungmann, J. Visschers, and M. J. J. Vrakking, Rev. Sci. Instrum. 80, 103105 (2009).

${ }^{9}$ M. N. R. Ashfold, N. H. Nahler, A. J. Orr-Ewing, O. P. J. Vieuxmaire, R. L. Toomes, T. N. Kitsopoulos, I. A. Garcia, D. A. Chestakov, S. M. Wu, and D. H. Parker, Phys. Chem. Chem. Phys. 8, 26 (2006).

${ }^{10}$ C. Vallance, M. Brouard, A. Lauer, C. S. Slater, E. Halford, B. Winter, S. J. King, J. W. L. Lee, D. E. Pooley, I. Sedgwick, R. Turchetta, A. Nomerotski, J. J. John, and L. Hill, Phys. Chem. Chem. Phys. 16, 383 (2014).

${ }^{11}$ R. Dörner, V. Mergel, O. Jagutzki, L. Spielberger, J. Ullrich, R. Moshammer, and H. Schmidt-Böcking, Phys. Rep. 330, 95 (2000).

${ }^{12}$ V. Stert, W. Radloff, C. P. Schulz, and I. V. Hertel, Eur. Phys. J. D 5, 97 (1999)

${ }^{13}$ K. Amini, S. Blake, M. Brouard, M. B. Burt, E. Halford, A. Lauer, C. S. Slater, J. W. L. Lee, and C. Vallance, Rev. Sci. Instrum. 86, 103113 (2015).

${ }^{14}$ J. H. Jungmann and R. M. A. Heeren, Rapid Commun. Mass Spectrom. 27, 1 (2013)

${ }^{15}$ S. K. Lee, F. Cudry, Y. F. Lin, S. Lingenfelter, A. H. Winney, L. Fan, and W. Li, Rev. Sci. Instrum. 85, 123303 (2014).

${ }^{16}$ F. J. Furch, S. Birkner, F. Kelkensberg, A. Giree, A. Anderson, C. P. Schulz, and M. J. J. Vrakking, Opt. Express 21, 22671 (2013).

${ }^{17}$ F. J. Furch, A. Giree, F. Morales, A. Anderson, Y. Wang, C. P. Schulz, and M. J. J. Vrakking, Opt. Express 24, 19293 (2016).

${ }^{18}$ X. Llopart, R. Ballabriga, M. Campbell, L. Tlustos, and W. Wong, Nucl. Instrum. Methods Phys. Res., Sect. A 581, 485 (2007).

${ }^{19}$ A. S. Tremsin, J. V. Vallerga, J. B. McPhate, O. H. W. Siegmund, and R. Raffanti, IEEE Trans. Nucl. Sci. 60, 578 (2013). 
${ }^{20}$ J. Vallerga, J. McPhate, A. Tremsin, and O. Siegmund, Nucl. Instrum. Methods Phys. Res., Sect. A 591, 151 (2008).

${ }^{21}$ J. Vallerga, R. Raffanti, A. Tremsin, J. McPhate, and O. Siegmund, J. Instrum. 6, C01049 (2011).

${ }^{22}$ A. S. Tremsin, J. V. Vallerga, O. H. W. Siegmund, and J. S. Hull, Proc. SPIE 5164, 113 (2003).

${ }^{23}$ R. Hong, A. Leredde, Y. Bagdasarova, X. Fléchard, A. García, P. Müller, A. Knecht, E. Liénard, M. Kossin, M. G. Sternberg, H. E. Swanson, and D. W. Zumwalt, Nucl. Instrum. Methods Phys. Res., Sect. A 835, 42 (2016).

${ }^{24}$ H. Hasegawa, A. Hishikawa, and K. Yamanouchi, Chem. Phys. Lett. 349, 57 (2001).
${ }^{25}$ See www.simion.com for the introduction of SIMION software and the simulation method in detail.

${ }^{26}$ C. Wu, G. Zhang, C. Wu, Y. Yang, X. Liu, Y. Deng, H. Liu, Y. Liu, and Q. Gong, Phys. Rev. A 85, 063407 (2012).

${ }^{27}$ S. J. King and S. D. Price, Int. J. Mass Spectrom. 272, 154 (2008).

${ }^{28}$ M. Alagia, P. Candori, S. Falcinelli, M. Lavollée, F. Pirani, R. Richter, S. Stranges, and F. Vecchiocattivi, J. Phys. Chem. A 113, 14755 (2009).

${ }^{29}$ M. Alagia, P. Candori, S. Falcinelli, M. Lavollée, F. Pirani, R. Richter, S. Stranges, and F. Vecchiocattivi, Phys. Chem. Chem. Phys. 12, 5389 (2010).

${ }^{30}$ S. Larimian, S. Erattupuzha, S. Mai, P. Marquetand, L. González, A. Baltuška, M. Kitzler, and X. Xie, Phys. Rev. A 95, 011404(R) (2017). 